\title{
A targeted salvage therapy with Brentuximab vedotin in heavily treated refractory or relapsed pediatric Hodgkin lymphoma patients before and after stem cell transplantation
}

\author{
Nurdan Taçyıldız' ${ }^{1}$ Hikmet Gülşah Tanyıldız ${ }^{1}$ Emel Ünal ${ }^{1}$, Handan Dinçaslan ${ }^{1}$, \\ Fikret Asarcıklı11 ${ }^{1}$, Başak Adaklı Aksoy ${ }^{1}$, Göksel Vatansever ${ }^{2}$, Gülsan Yavuz ${ }^{1}$ \\ Divisions of ${ }^{1}$ Pediatric Oncology and ${ }^{2}$ Department of Pediatrics, Ankara University Faculty of Medicine, Ankara, Turkey. \\ E-mail:g_oktay4910@yahoo.com \\ Received: 31 st July 2018, Revised: 14th February 2019, 4th March 2019, Accepted: 11th March 2019
}

\begin{abstract}
SUMMARY: Taçyıldız N, Tanyıldız HG, Ünal E, Dinçaslan H, Asarcıklı F, Adaklı Aksoy B, Vatansever G, Yavuz G. A targeted salvage therapy with Brentuximab vedotin in heavily treated refractory or relapsed pediatric Hodgkin lymphoma patients before and after stem cell transplantation. Turk J Pediatr 2019; 61: 671-676.
\end{abstract}

Hodgkin's lymphoma (HL) is highly curable disease in its early stages, but in advanced stages, it presents a dilemma when it becomes refractory or relapses after several rounds of chemotherapy. Brentuximab vedotin (BV) is an antibody-drug conjugate that targets the tumor necrosis receptor family protein member CD30 positive malignancies via an anti-CD30 monoclonal antibody linked to monomethyl auristatin-E. In adult and pediatric studies, it has been shown to be an effective salvage therapy for primary refractory $\mathrm{HL}$ or relapse after autologous stem cell transplant (ASCT).

Between July 2012 and August 2017, we administered BV (1.8 mg/m² every three weeks; 12 cycles totally) with doxorubucin, vinblastin, dacarbazine (AVD), rituximab + ifosfamide + carboplatin + etoposide (RICE), or bendamustine combination treatment in pediatric HL patients, who were previosuly treated for refractory or relapsed advanced stage HL before (seven patients) or after (one patient) ASCT in our center. After eight BV courses, one patient was able to undergo match unrelated donor (MUD) SCT. Another seven pediatric HL patients, who were not able to go into remission with any other classical HL chemotherapy protocols, received 4-6 courses of BV-AVD and/or RICE/bendamustine. All were able to undergo ASCT after negative positron emission tomography (PET) imaging results. After ASCT, we switched to BV as consolidation therapy until a total of 12 cycles was completed. Patients went into remission after a median 34 (range: 12-42) months from the start of BV treatment. BV is an encouraging, well- tolerated, and effective targeted therapy especially when combined with AVD or when alternated with another targeted therapy combination, including RICE, when needed.

Key words: Hodgkin lymphoma, brentuximab vedotin.

Current treatment modalities can cure up to $95 \%$ of patients with the classical Hodgkin's lymphoma (HL); however, the treatment becomes a big dilemma when the HL is refractory, or relapsed disease has reached advanced stage of chemotherapy courses causing approximately $5 \%-10 \%$ of patients to require further treatment options. However, brentuximab (BV) is an anti-CD30 antibody conjugated via protease-cleavable linker to the

This manuscript was presented as an oral presentation at the 21st National Cancer Congress, 22-26 April 2015, Antalya, Turkey. 
potent anti-microtubule agent monomethyl auristatin-E (MMAE). Following binding to $\mathrm{CD} 30, \mathrm{BV}$ vedotin is rapidly internalized and transported to lysosomes where MMAE is released and binds to tubulin, leading to cell cycle arrest and apoptosis. BV has been approved for the treatment of relapse refractory disease or consolidation after autologous transplantation in HL. ${ }^{1-4}$ Despite BV's efficacy, there is still a scarcity of studies that focus on Hodgkin's lymphoma (HL) in children. Typically, it has been observed in Phase I/II-level studies that BV may serve as a frontline agent for high-risk pediatric $\mathrm{HL}$ patients. ${ }^{5}$ With reference to children with relapsed-refractory $(\mathrm{RR}) / \mathrm{HL}$, the primary goal is to enable autologous or allogeneic stem cell transplantation after achieving salvage chemotherapy induced remission with fewer side effects in these patients. In this study, we report the experience of using highdose chemotherapy with autologous stem cell transplantation (ASCT), which remains the current standard of treatment for young patients with HL who are in the first relapse period or in those who are refractory to firstline treatment. We used BV in combination with doxorubucin, vinblastin, dacarbazine (AVD), bendamustine, or rituximab + ifosfamide + carboplatin + etoposide (RICE) in eight RR/HL pediatric patients. The use of BV provided a bridge until it was time for SCT.

\section{Material and Methods}

We retrospectively evaluated our BV experience in patients diagnosed with HL between 2012 and 2017. In our clinic, BV has been used for pediatric HL patients who underwent early relapse or were refractory to primary treatment or relapsed after ASCT since 2012. Early relapse was considered as occuring between three and 12 months after the end of therapy. AVD with bleomycin (AVBD) was used as standard therapy for initial treatment, whereas BV combined AVD, RICE, or bendamustine was used as second-line therapy after relapse in HL patients. However, our first experince with BV as a targeted treatment was in the first patient who relapsed after ASCT because BV was not available at that time for refractory or relapse disease in our country. Since the first patient's pathological study showed CD20 positivity, while the others had been CD20 negative, we were able to use antiCD20 treatment only in this patient. Thus, BV was used on eight pediatric patients who relapsed just after chemotherapy or ASCT or were refractory to the treatment and whose tumor tissues were CD30 positive. A $1.8 \mathrm{mg} /$ $\mathrm{kg}$ dose of $\mathrm{BV}$ was administered at intervals of three weeks with an infusion period longer than $30 \mathrm{~min}$ before and after SCT for at least 12 cycles as consolidation therapy. For BV's safety and suitability, the National Cancer Institute (NCI) Common Toxicology Criteria were taken into consideration before each BV cycle (23). Common adverse effects of $\mathrm{BV}$, such as nausea, vomiting, fatigue, myalgia, constipation, and / or itching were recorded, and side effects related to $\mathrm{BV}$ were recorded. Any adverse effects, which would limit the use of BV, such as chronic kidney failure, seizures, demyelinating neuropathy, or myelosuppression were evaluated prior to drug use. Patients' responses to chemotherapy before and after BV were determined by physical examinations, laboratory analyses, and radiologic imaging (ultrasonography, computed tomography, and positron emission tomography $[\mathrm{PET}])$. Clinical, radiological, and nuclear medicine evaluation (PET) were obtained after every two cycles of chemoterapy. The primary refractory disease was assessed as relapse or failure to achieve complete remission during the first three months of first- line treatment. The study was reviewed and approved by an Ethics Committee (IRB:03113-13). The authors report no conflicts of interest and any financial relationship.

\section{Results}

The median age of the eight participating patients' was $14(6-18)$ years. The ratio of males to females was $7 / 1$. Histopathological diagnoses of patients consisted of two findings: (1) mixed celluler $(n=6)$ and (2) nodular sclerosis $(n=2)$. Five patients were determined to have stage IVB, and three patients had stage IIIB. Three patients received chemotherapy in combination with BV for the primary refractory disease. Moreover, three patients with early relapse and two patients with relapsed HL after ASCT received chemotherapy in combination with BV. Except for the first patient, the 
number of BV administrations was between 4 and 6 cycles in most of the patients before they underwent ASCT. After transplantation and in order to resume control of the disease, we continued BV up to 12 cycles. In this way, four of our patients went into remission after the second BV course. Moreover, three and one patient(s) went into remission after the fourth and eigth BV course, respectively. One patient, who received BV after his disease relapsed 12 months after ASCT (conditioning regimen containing total body irradiation [TBI] and melphalan), was the first pediatric patient who received BV in our center and country. He was in remission with negative PET result after six courses of BV-RICE (due to his CD20 positivity in tumor tissues) and two courses of BV-bendamustine. After eight BV courses, he was able to undergo match unrelated donor (MUD)-SCT. Donor leukocyte infusion (DLI) was administered to the patient who relapsed again 12 months after MUD. Following the DLI, he developed skin and gastrointestinal graft versus host disease (GIS- GVHD). Moreover, two more courses of BV in conjunction with bendamustine were administered to the patient after the DLI. PET-based involvement in one patient, who received two additional courses of BV and bendamustine in total after DLI, completely disappeared. We decreased the dosage from 1.8 to $1.2 \mathrm{mg} / \mathrm{kg}$ after $10 \mathrm{BV}$ cycles and stopped BV on the $12^{\text {th }}$ application because of neurotoxicity. The patient was still attending follow-ups with remission at the $36^{\text {th }}$ month after BV treatment initiation. The second patient underwent ASCT because of refractory disease following the pre-emptive TBI and melphalan regimen. Moreover, BV treatment of eight cycles, which had been initiated before the transplant, was resumed during follow-up. This patient died from lymphoproliferative disease secondary to EBV in the $12^{\text {th }}$ month after BV initiation. The third patient, who relapsed nine months after autologous transplantation, received $\mathrm{BV}$ and bendamustine in combination with the aim of remission before undergoing ASCT. However, remission obtained after six cycles caused the disease to relapse again, and the patient died from refractory disease. The other five $\mathrm{RR} / \mathrm{HL}$ patients underwent ASCT following $\mathrm{BV}$ in combination with AVD. The patients were followed while in remission for 42,40 ,
32, 32, and 24 months from BV treatment initiation, respectively (Table I). Fatigue was the most common side effect, and because of neurotoxicity, the BV dose had to be reduced in one patient.

\section{Discussion}

The cure rate of $\mathrm{HL}$ in pediatric patients is $>95 \%$, owing to recent advances in treatment options. ${ }^{6}$ However, early relapse or treatment of primary refractory disease is still an important problem. With the associated risks of secondary cancer and cardiopulmonary toxicity, new modalities for minimizing toxicity in these patients are targeted similarly. Nuclear factor (NF)-kB and CD30 are also being identified as potential biomarkers in HL patients, suggesting that anti-CD30 could be used as a targeted treatment agent. ${ }^{7,8}$

In Locatelli's ${ }^{5}$ study, BV was shown to be effective in pediatric patients with poor prognostic HL. BV has manageable toxicity and is associated with clinically meaningful responses in pediatric patients with relapsed or refractory HL. The use of BV enabled the investigators to provide a bridge to SCT for some patients. ${ }^{9}$

In this context, it has been shown that targeted treatment agents, such as CD30, are important for treating early relapsed or refractory HL cases before transplantation in order to achieve remission after the transplant. This shows that the use of valuable targeted treatment agents is important as a consolidation treatment for treatment of early relapsed or refractory cases of HL patients. In this group of patients, a salvage (rescue) and high-dose chemotherapy regimen followed by ASCT constitutes the standard treatment approach. Nevertheless, under these circumstances, $50 \%$ of patients experienced relapse and a certain decline in the quality of life during follow-up. The aim is to have a consolidation treatment that will prevent the relapse of the disease following ASCT. ${ }^{10-14}$

Two randomized trials showed a significant improvement in progression-free survival after ASCT, and several large studies have shown that this procedure can provide a cure in roughly $50 \%$ of the patients undergoing ASCT. 


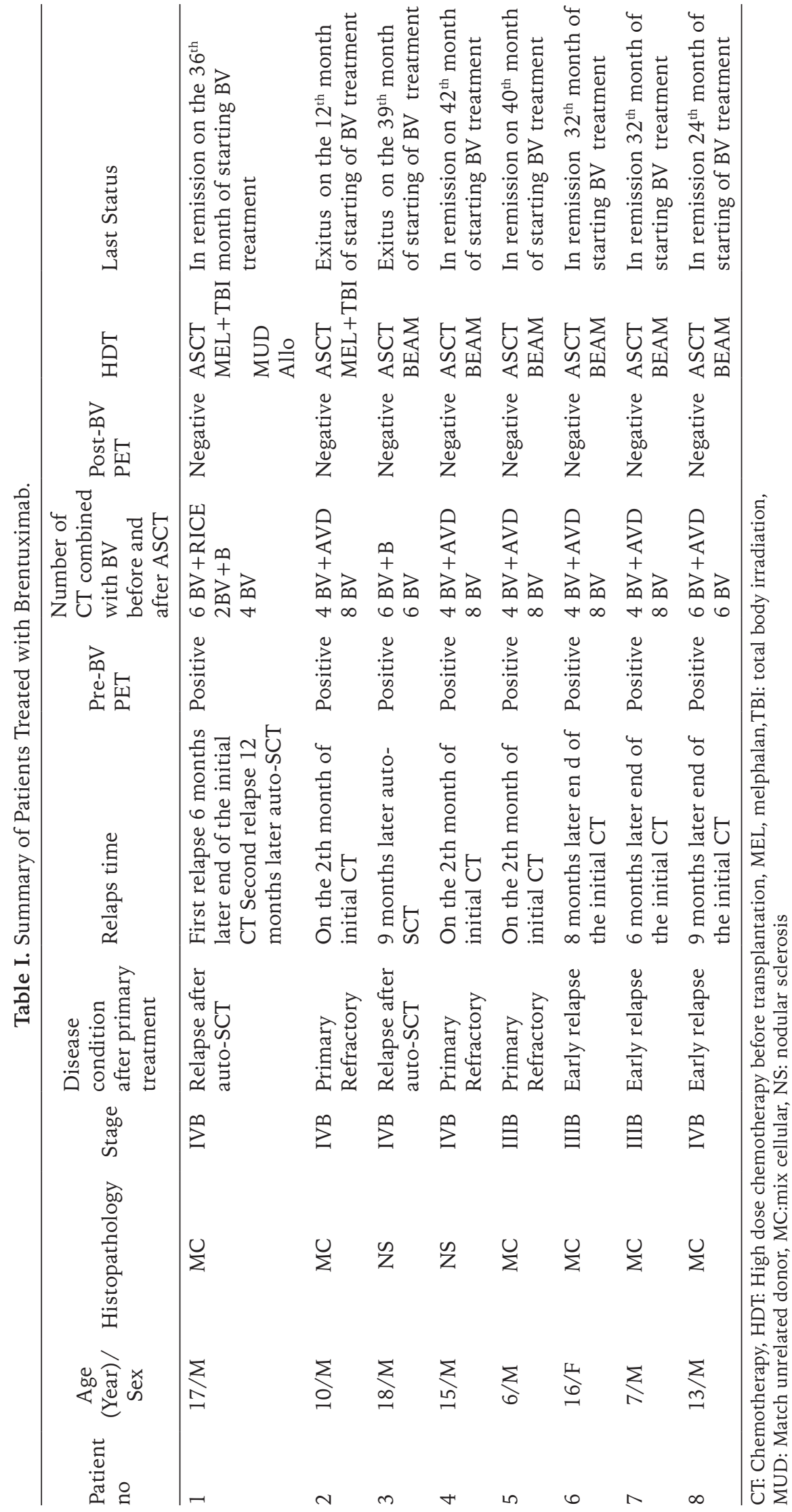


Various treatment strategies for improving outcomes after ASCT have been investigated, including PET-adapted approaches, intensification of the conditioning regimen, radiation before and after transplantation, and consolidation therapy after transplantation. ${ }^{5}$ Early consolidation with BV after ASCTrelated improvements in progression free survival showed substantial efficacy in patients, including an objective response rate of $75 \%$ and complete remission rate of $34 \%$ in a pivotal phase 2 study of patients with CD30-positive HL in whom high-dose therapy and ASCT had been ineffective; longer-term follow-up showed a median overall survival of 40.5 months. As a targeted therapy with a low frequency of severe hematological toxic effects, use of BV might provide a unique opportunity to deliver pre-emptive therapy after ASCT. ${ }^{9,12,15}$

This study is quite promising as it reflects the first experience with $\mathrm{BV}$ in children with advanced stage RR/HL cases and positive results with respect to the use of $\mathrm{BV}$, especially in early relapsed or refractory HL. In our study, six patients with early relapsed or primary refractory disease that have been treated with BV combined with AVD were able to go into remission. All of the six patients were able to undergo ASCT. The other two patients relapsed after ASCT. They were successfully treated with BV. After that successful treatment was accomplished, bridging BV therapy in one of them allowed him to undergo ASCT. He has survived so far, but unfortunately the second one died after the sixth BV cycle due to relapse/refractory disease. The outcome of the results of our single-center retrospective study demonstarted that $\mathrm{BV}$ is being considered as a safe agent in primary refractory and relapsed pediatric HL cases who are able to undergo ASCT. In the literature concerning adult cases, we have not encountered any adverse effects, such as chronic kidney failure, seizures, demyelinating neuropathy, and/or myelosuppression, that have been reported in association with BV and could limit BV's use. ${ }^{16,17}$ Common adverse BV-associated effects are nausea, vomiting, fatigue, myalgia, constipation, and/or itching. However, the most common side effect observed in this study was fatigue. Neuropathy was associated with
BV use were encountered in the first patient who underwent long-term chemotherapy and transplantation. Because of BV-induced neurotoxicity, we decreased the dosage from 1.8 to $1.2 \mathrm{mg} / \mathrm{kg}$. Since neurotoxicity was progressing, we stopped the $\mathrm{BV}$ treatment at the followup. As there is limited data on its use in children, an administration plan has been proposed based on the adult literature (1.8 $\mathrm{mg} / \mathrm{kg}$ at intervals of three weeks). ${ }^{16,18}$ Recently, a study supporting our practice was published by Flerlage et al. ${ }^{19}$ who reported that BV administration to children is safe at a dose of $1.8 \mathrm{mg} / \mathrm{kg}$ at intervals of three weeks. However, we still have to be careful about neurotoxicity, especially during repeated BV cycles. It was observed that PET involvement disappeared, and remission was clinically and radiologically achieved in five patients after the fourth course of $\mathrm{BV}$, in two patients after the second BV course, and in one patient after the eigth BV course. As rapid remission is induced by $\mathrm{BV}$, we believe that $\mathrm{BV}$ is an effective drug for relapsed and primary refractory patients. ${ }^{16,17}$ It is also effective for controlling the disease before transplantation and maintaining disease-free control after transplantation. Other studies similarly report that good outcomes are obtained in controlling of disease with BV administration. ${ }^{20-22}$ We adminsistered BV after transplantation for maintainance of therapy in all patients, and they are still in remission with the exception of two patients. We observed that it had a positive effect on the improvement of one of our patient's GVHD symptoms after alloSCT. In the literature, it has been reported that BV has a positive role in the elimination of alloreactive CD30 + donor $\mathrm{T}$ cells, thereby, contributing to the improvement of GVHD symptoms. ${ }^{23}$ It is true that outcomes and experience from additional studies, which would reveal the positive and negative effects of BV and include many more pediatric patients, are needed. We still believe that our results will guide clinicians on BV's use since there are a lack of studies regarding BV's use in pediatric patients. In conclusion, BV is a safe salvage treatment agent with limited adverse effects for pediatric patients with refractory or relapsed advanced-stage HL before and after SCT. 


\section{REFERENCES}

1. Skarbnik AP, Pro B. Heads or tails? Choosing a salvage therapy for relapsed/refractory Hodgkin lymphoma. Expert Rev Hematol 2013; 6: 1-3.

2. Gualberto, A. Brentuximab Vedotin (SGN-35), an antibody-drug conjugate for the treatment of CD30positive malignancies. Expert Opin Investig Drugs 2012; 21: 205-216.

3. Bair SM, Strelec L, Nagle SJ, et al. Outcomes of patients with relapsed/refractory Hodgkin lymphoma progressing after autologous stem cell transplant in the current era of novel therapeutics: A retrospective analysis. Am J Hematol 2017; 92: 879-884.

4. Bonthapally V, Yang H, Ayyagari R, et al. Brentuximab vedotin compared with other therapies in relapsed/ refractory Hodgkin lymphoma post autologous stem cell transplant: median overall survival meta-analysis. Curr Med Res Opin 2015; 31: 1377-1389.

5. Locatelli F, Koerholz-Mauz C, Neville K, et al. Brentuximab vedotin for paediatric relapsed or refractory Hodgkin's lymphoma and anaplastic largecell lymphoma: a multicentre, open-label, phase $1 / 2$ study. Lancet Haematol 2018; 5: e450-e461.

6. Kumar A, Casulo C, Yahalom J, et al. Brentuximab vedotin and AVD followed by involved-site radiotherapy in early stage, unfavorable risk Hodgkin lymphoma. Blood 2016; 128: 1458-1464.

7. Nagpal P, Akl MR, Ayoub NM, et al. Pediatric Hodgkin lymphoma: biomarkers, drugs, and clinical trials for translational science and medicine. Oncotarget 2016; 7: 67551-67573.

8. Horie R, Watanabe T, Morishita Y, et al. Ligandindependent signaling by overexpressed CD30 drives NF-kappaB activation in Hodgkin-Reed-Sternberg cells. Oncogene 2002; 21: 2493-2503.

9. Younes A, Gopal AK, Smith SE, et al. Results of a pivotal phase II study of Brentuximab vedotin for patients with relapsed or refractory Hodgkin's lymphoma. J Clin Oncol 2012; 30: 2183-2189.

10. Schellong G, Dörffel W, Claviez A, et al; DAL/ $\mathrm{GPOH}$. Salvage therapy of progressive and recurrent Hodgkin's disease: results from a multicenter study of the pediatric DAL/GPOH-HD study group. J Clin Oncol 2005; 23: 6181-6189.

11. Satwani P, Jin Z, Martin PL, et al. Sequential myeloablative autologous stem cell transplantation and reduced intensity allogeneic hematopoietic cell transplantation is safe an feasible in children, adolescents and young adults with poor-risk refractory or recurrent Hodgkin and non-Hodgkin lymphoma. Leukemia 2015; 29: 448-455.
12. Moskowitz $\mathrm{CH}$, Nademanee $\mathrm{A}$, Masszi $\mathrm{T}$, et al AETHERA Study Group. Brentuximab vedotin as consolidation therapy after autologous stem-cell transplantation in patients with Hodgkin's lymphoma at risk of relapse or progression (AETHERA): a randomised, doubleblind, placebo-controlled, phase 3 trial. Lancet 2015; 385: 1853-1862.

13. Zinzani PL, Sasse S, Radford J, Shonukan O, Bonthapally V. Experience of brentuximab vedotin in relapsed/refractory Hodgkin lymphoma and relapsed/ refractory systemic anaplastic large-cell lymphoma in the Named Patient Program: Review of the literature. Crit Rev Oncol Hematol 2015; 95: 359-369.

14. Majhail NS, Weisdorf DJ, Defor TE, et al. Long-term results of autologous stem cell transplantation for primary refractory or relapsed Hodgkin's lymphoma. Biol Blood Marrow Transplant 2006; 12: 1065-1072.

15. Gopal AK, Chen R, Smith SE, et al. Three-year follow up data and characterization of long term remissions from an ongoing phase 2 study of brentuximab vedotin in patients with relapsed or refractory hodgkin lymphoma. Blood 2013; 21: 4382.

16. Tsirigotis $\mathrm{P}$, Danylesko I, Gkirkas $\mathrm{K}$, et al Brentuximab vedotin in combination with or without donor lymphocyte infusion for patients with Hodgkin lymphoma after allogeneic stem cell transplantation. Bone Marrow Transplant 2016; 51: 1313-1317.

17. Salihoglu A, Elverdi T, Karadogan I, et al. Brentuximab vedotin for relapsed or refractory Hodgkin lymphoma: experience in Turkey. Ann Hematol 2015; 94: 415420.

18. Younes A, Bartlett NL, Leonard JP, et al. Brentuximab vedotin (SGN-35) for relapsed CD30-positive lymphomas. N Engl J Med 2010; 363: 1812-1821.

19. Flerlage JE, Metzger ML, Wu J, Panetta JC. Pharmacokinetics, immunogenicity, and safety of weekly dosing of brentuximab vedotin in pediatric patients with Hodgkin lymphoma. Cancer Chemother Pharmacol 2016; 78: 1217-1223.

20. Rothe A, Sasse S, Goergen H, et al. Brentuximab vedotin for relapsed or refractory CD30+ hematologic malignancies: the German Hodgkin Study Group experience. Blood 2012; 120: 1470-1472.

21. Gibb A, Jones C, Bloor A, et al. Brentuximab vedotin in refractory $\mathrm{CD} 30$ + lymphomas: a bridge to allogeneic transplantation in approximately one quarter of patients treated on a Named Patient Programme at a single UK center. Haematologica 2013; 98: 611-614.

22. Zinzani PL, Viviani S, Anastasia A, et al. Brentuximab vedotin in relapsed/refractory Hodgkin's lymphoma: the Italian experience and results of its use in daily clinical practice outside clinical trials. Haematologica 2013; 98: 1232-1236.

23. Chen $\mathrm{YB}$, McDonough $\mathrm{S}$, Hasserjian $\mathrm{R}$, et al Expression of $\mathrm{CD} 30$ in patients with acut graftversus-host disease. Blood 2012; 120: 691-696. 